\title{
Hidden Charge States in Soft-X-Ray Laser-Produced Nanoplasmas Revealed by Fluorescence Spectroscopy
}

\author{
L. Schroedter, ${ }^{1}$ M. Müller, ${ }^{2}$ A. Kickermann, ${ }^{1}$ A. Przystawik, ${ }^{1}$ S. Toleikis, ${ }^{1}$ M. Adolph, ${ }^{2}$ L. Flückiger, ${ }^{2}$ \\ T. Gorkhover, ${ }^{2}$ L. Nösel, ${ }^{2}$ M. Krikunova, ${ }^{2}$ T. Oelze, ${ }^{2}$ Y. Ovcharenko, ${ }^{2}$ D. Rupp, ${ }^{2}$ M. Sauppe, ${ }^{2}$ D. Wolter, ${ }^{2}$ \\ S. Schorb, ${ }^{3}$ C. Bostedt, ${ }^{3}$ T. Möller, ${ }^{2}$ and T. Laarmann ${ }^{1,4, *}$ \\ ${ }^{1}$ Deutsches Elektronen-Synchrotron DESY, Notkestrasse 85, 22607 Hamburg, Germany \\ ${ }^{2}$ Institut für Optik und Atomare Physik, Technische Universität Berlin, Eugene-Wigner-Building EW 3-1, \\ Hardenbergstrasse 36, 10623 Berlin, Germany \\ ${ }^{3}$ SLAC National Accelerator Laboratory, P.O. Box 20450, Stanford, California 94309, USA \\ ${ }^{4}$ The Hamburg Centre for Ultrafast Imaging CUI, Luruper Chaussee 149, 22761 Hamburg, Germany
}

(Received 18 December 2013; published 5 May 2014)

\begin{abstract}
Highly charged ions are formed in the center of composite clusters by strong free-electron laser pulses and they emit fluorescence on a femtosecond time scale before competing recombination leads to neutralization of the nanoplasma core. In contrast to mass spectrometry that detects remnants of the interaction, fluorescence in the extreme ultraviolet spectral range provides fingerprints of transient states of high energy density matter. Spectra from clusters consisting of a xenon core and a surrounding argon shell show that a small fraction of the fluorescence signal comes from multiply charged xenon ions in the cluster core. Initially, these ions are as highly charged as the ions in the outer shells of pure xenon clusters with charge states up to at least $11+$.
\end{abstract}

DOI: 10.1103/PhysRevLett.112.183401

PACS numbers: $36.40 . \mathrm{Gk}, 32.50 .+\mathrm{d}, 52.25 .0 s, 79.77 .+\mathrm{g}$

Structure and dynamics of matter interacting with strong laser pulses raised a lot of interest in the last decade, because new experimental regimes became accessible that push investigations to new boundaries: (i) $\mathrm{x}$ rays provide atomic spatial resolution in diffraction experiments and photoionize inner-shell electrons, giving element-specific information; (ii) a high photon flux allows us to collect a scattering signal from small, dilute, or nonperiodic systems and to deposit a large number of $\mathrm{x}$-ray photons per atom generating extreme states of matter far from equilibrium; (iii) ultrashort light pulses make it possible to study phase transitions and structural changes in real time. Each of these aspects enables novel research but even more possibilities for experiments open up if they are all available simultaneously as in the case of short-wavelength free-electron lasers (FELs) [1-4]. A proposed revolutionary application of FELs is the imaging of a single molecule in a single light pulse [5]. Coherent diffraction imaging promises to reveal the structure of molecules which cannot be prepared in crystalline form. Time-resolved pump-probe schemes enable experiments which are often advertised as recording "molecular movies." However, a severe challenge is the damage done to the molecule by the light pulse. Besides elastic scattering by bound electrons, strong ionization takes place and the molecule is destroyed. It is an open question whether sufficient scattering signal can be detected before the molecule disintegrates. One possible way to alleviate this problem is adding a tamper layer around the molecule which slows down the expansion [6]. At this point, cluster science comes into play. Clusters can be used as nanoscopic model systems to measure how much energy is absorbed. Because they are surrounded by vacuum, no energy can dissipate out of the system. In particular rare-gas clusters can be easily generated by expanding gas through a nozzle. Their relatively simple structure makes them accessible via theoretical calculations $[7,8]$. Last but not least, it is possible to test the effect of tamper layers, as so-called core-shell clusters can be generated, with a core consisting of one element surrounded by a shell consisting of another element $[9,10]$.

In this Letter we report FEL-induced fluorescence spectra from $\mathrm{Xe}$, Ar, and Xe-core-Ar-shell clusters in the extreme ultraviolet (XUV) spectral range. The experiments are conducted at the soft X-ray free-electron laser in Hamburg (FLASH) [1]. The excitation wavelength of $13.5 \mathrm{~nm}$ is resonant with the $4 d$ electron shell of Xe. The experimental setup shown in Fig. 1 is described in detail in [11]. For the current investigation a few modifications have been made. These concern mainly the fluorescence detection that will be described in the following. Briefly, a multilayer mirror with a focal length of $450 \mathrm{~mm}$ reflects the FEL beam at almost normal incidence which results in a beam waist of $d \approx 2.5 \mu \mathrm{m}$ (full width at half maximum). At typical pulse durations of $150 \mathrm{fs}$ and an average pulse energy above $100 \mu \mathrm{J}$ the power density exceeds $10^{15} \mathrm{~W} / \mathrm{cm}^{2}$ with a typical shot-to-shot fluctuation of 30\%-40\% [1]. This causes massive absorption of at least 17 photons per Xe atom [12]. The pulse intensity in the interaction region can be changed by moving the focus along the FEL axis. The cluster beam intersects with the 


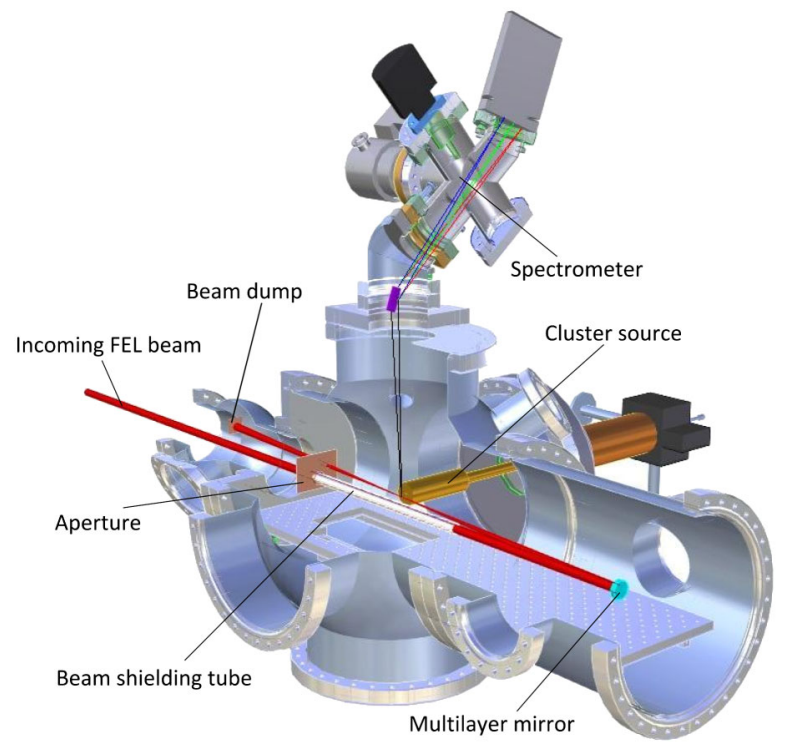

FIG. 1 (color online). Experimental setup for detecting cluster fluorescence. Before the FEL is refocused with a movable multilayer mirror onto the cluster beam, it passes through an aperture and a tube ensuring that no background ions are generated. Both the cluster beam and the FEL beam intersect in the spectrometer focus defined by the toroidal grating.

FEL beam almost perpendicularly. The interaction results in the emission of fluorescence photons from multiply charged ions. For energy-resolved photon detection a grazing incidence spectrometer is mounted perpendicular to both the FEL beam and the cluster beam. A toroidal diffraction grating with variable line spacing and an average of 450 lines $/ \mathrm{mm}$ diffracts and focuses the fluorescence onto an XUV-sensitive CCD camera. The flat-field spectrometer detects fluorescence in a wide wavelength range from 10 to $76 \mathrm{~nm}$. Its overall detection efficiency is about $1 \times 10^{-7}$ including acceptance angle, grating efficiency, and camera sensitivity according to simulations. The measured resolution is about $0.2 \mathrm{~nm}$ over the covered spectral range. Pure clusters were produced by adiabatic expansion of rare gases through a pulsed $100 \mu \mathrm{m}$ conical nozzle with a half-opening angle of $15^{\circ}$. The average cluster size $\bar{N}$ was controlled by adjusting the stagnation pressure [13]. For Xe, $\bar{N}$ was varied between about 260 and 19000 atoms. Average cluster sizes of $\mathrm{Ar}$ were varied between about 40 and 6000 atoms. The coreshell clusters are prepared by coexpansion of a gas mixture. With the cluster source used in this experiment well defined, core-shell structures can be produced by expanding a mix of $1 \%-5 \% \mathrm{Xe}$ in $\operatorname{Ar}[14,15]$. A core of Xe atoms nucleates on the inside surrounded by Ar atoms at the surface of the clusters due to differences in binding energies and melting points of the constituents. The thickness of the Ar surface layer depends sensitively on the Xe concentration which is $2 \%$ in the present experiments. Adiabatic expansion at room temperature and 6.5 bar stagnation

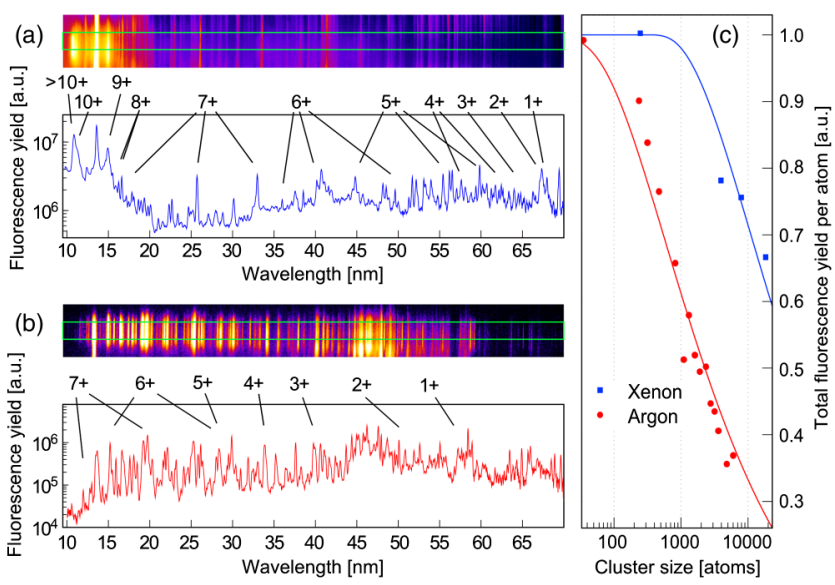

FIG. 2 (color online). Typical fluorescence spectra of pure Xe clusters (a) and pure Ar clusters (b) comprising 4100 and 400 atoms, respectively. The data are recorded with an x-ray camera at a FEL intensity of approximately $2 \times 10^{15} \mathrm{~W} / \mathrm{cm}^{2}$ and plotted in logarithmic scale. The same line out of the CCD chip (green rectangle) is evaluated for each spectrum throughout the present work. Charge states have been assigned to more than 100 fluorescence lines $(\sim 80 \%)$ and a characteristic selection is given. Total fluorescence yield per atom as a function of cluster size is shown in (c).

pressure generates clusters of about $400 \mathrm{Ar}$ atoms with an embedded core of about $80 \mathrm{Xe}$ atoms.

A typical fluorescence spectrum of pure $\mathrm{Xe}$ and $\mathrm{Ar}$ clusters is plotted in Figs. 2(a) and 2(b), respectively. Here, an exposure time of $2 \mathrm{~min}$ was used, equal to an accumulation over 1200 FEL shots. For each exposure time, we record a background image, where the FEL enters the chamber but the cluster source was turned off. The background image was subtracted from each spectrum to ensure that stray light of the FEL hitting surfaces within the chamber did not have an effect on the spectra. The raw data is integrated vertically in a narrow region, as indicated by the green rectangle in Fig. 2, to retrieve a spectrum. This region represents the dimension along the FEL beam axis which can be properly focused by the spectrometer grating and which is smaller than the Rayleigh length $z_{0}=1.1 \mathrm{~mm}$ of the focusing optics. For a quantitative analysis of the spectra, wavelength-dependent efficiency of the spectrometer was taken into account. This includes both the transmission of the grating and the sensitivity of the x-ray CCD. The assignment of charge states is possible by searching for correlations in the FEL power density dependence of fluorescence line intensities in comparison with available data from atomic spectra [16-18]. In the experiments on pure Xe clusters, charge states up to at least $11+$ were detected. The Ar cluster fluorescence reveals maximum charge states of up to $7+$. The detected Ar charge states are lower due to both the higher ionization potential of Ar and the difference in the atomic photoabsorption cross section of $24.7 \mathrm{Mb}$ for Xe compared with $1.4 \mathrm{Mb}$ for $\mathrm{Ar}$ at a wavelength of $13.5 \mathrm{~nm}$. 
XUV fluorescence is known as a powerful tool to obtain information on electron density and temperature of plasmas generated from solid samples where the plasma environment induces line broadening, shifts, or population changes [19]. However, these effects are too small to be discernible in our experiment. The radiative decay in a transient cluster nanoplasma is affected by two competing dynamic processes of (a) nonradiative recombination and (b) complete disintegration of the clusters. The latter is driven by Coulomb explosion of the ionic shell and hydrodynamic expansion of the nanoplasma core; see, e.g., [20] and references therein. Under the present experimental condition a cluster plasma comprising a few thousand atoms doubles its radius within 1-2 ps [7]. In both cases of pure Ar and Xe clusters interacting with strong fs FEL pulses, fluorescence is predominantly emitted from a shell of highly charged ions on the surface of the cluster during the disintegration when the number density has already significantly decreased. The fraction of surface atoms decreases with increasing cluster size as can be seen in the reduced fluorescence yield per atom in Fig. 2(c), while the spectral distribution hardly changes.

To push the experimental sensitivity toward processes that occur inside the cluster ion core on the sub-ps time scale when the core is still close to its initial geometry, we performed complementary fluorescence experiments on Xe-core-Ar-shell clusters. In the past, various experimental and theoretical studies have been performed on FELinduced ultrafast nanoplasma dynamics [7,8,21-26]. In most cases ion spectra were evaluated that have been recorded with time-of-flight (TOF) techniques. By choosing adequate static electric extraction fields, virtually all ions which result from the total disintegration of the cluster can be collected and guided to the detector. The method allows us to determine the charge state of ions by measuring their flight time from the interaction region to the detector. Even though the photoionization probability of the Xe core is significantly larger than that of the surrounding Ar shell, high Xe charge states from the coreshell cluster are absent in TOF spectra [27]. This gives experimental evidence that electrons are efficiently transferred to the cluster center. In agreement with theoretical work [28], the quasifree electrons that are ionized from individual atoms but have not left the cluster as a whole recombine to a large extent with ions in the cluster core. From the detection of remnants of the FEL-cluster interaction, it is concluded that indeed the positive charge is concentrated on a shell on the outside of the cluster, which explodes off due to the Coulomb forces, whereas the cluster core expands hydrodynamically at somewhat slower speed [29].

It is important to note that using mass spectrometry has significant implications: microseconds pass from the creation of the ions to their reaching of the detector, enough time for postinteraction processes to take place such as delayed electron emission during the cluster expansion. Second, the electrostatic fields of a few $\mathrm{kV} / \mathrm{cm}$ may alter the experimental observation [30]. Finally, space charge in dense cluster beams may affect the measured kinetic energy distributions, leading to an overestimate of the absorbed energy. Nonetheless, as ions are initially created and recombine in the evolving nanoplasma, there is also a chance of their immediate decay emitting a fluorescence photon [31], while the cluster is much closer to its unperturbed geometry. In other words, the time-integrated fluorescence signal also contains fingerprints from the earliest stage of the FEL cluster interaction. The welldefined radial distribution of different elements in Xe-core and Ar-shell clusters allows to disentangle directly the radiative decay of the Coulomb exploding cluster ion surface (Ar) from fluorescence that stems from the center of the Xe nanoplasma core because the characteristic shortwavelength fluorescence appears in different spectral ranges. $\mathrm{Ar}^{7+}$ predominantly emits at wavelengths longer than $12 \mathrm{~nm}$, whereas $\mathrm{Xe}^{q+}$ with $q>10+$ shows dominant fluorescence below $12 \mathrm{~nm}$. Most importantly, by analyzing the characteristic radiative transitions of $\mathrm{Xe}$ ions, we gain direct insight into the charge states that are initially created upon resonant excitation of the Xe core by the FEL. This information is hidden and not observable in TOF ion spectra. We note that our experimental parameters as well as the beam geometry and focusing optics are virtually the same as those in the ion TOF spectroscopic studies of coreshell clusters [27]. Nevertheless, in the present fluorescence experiment the highest observed charge state is slightly higher than those observed in previous mass spectra due to the higher FEL fluence (longer pulses) and therefore higher deposited energy. Notwithstanding these differences the application of fluorescence spectroscopy provides novel, complementary information on the ionization state of composite clusters.

Figure 3 shows a comparison between fluorescence spectra of core-shell clusters and pure Ar clusters. The relative line intensities vary between both spectra, but most lines which appear in the core-shell spectrum are also present in the pure Ar spectrum. This means that the vast majority of the spectrum consists of fluorescence from $\mathrm{Ar}$ ions, which were situated in the cluster shell. The evaluation of the relative change in line intensities is shown in the inset of Fig. 3. It turns out that the Ar charge state distribution shifts to higher values for core-shell clusters compared to pure Ar clusters due to the higher absorption and ionization cross section of the Xe cluster ion core. More importantly, Xe-core-Ar-shell clusters emit at short wavelengths between 10.5 and $12 \mathrm{~nm}$. This signal is absent in pure Ar clusters as can be seen. The wavelength range in which additional transitions appear is presented in Fig. 4. We assign the fluorescence at short wavelength to the $\mathrm{Xe}$ ion core because it coincides with the most prominent emission lines in the pure Xe spectra. To corroborate this 


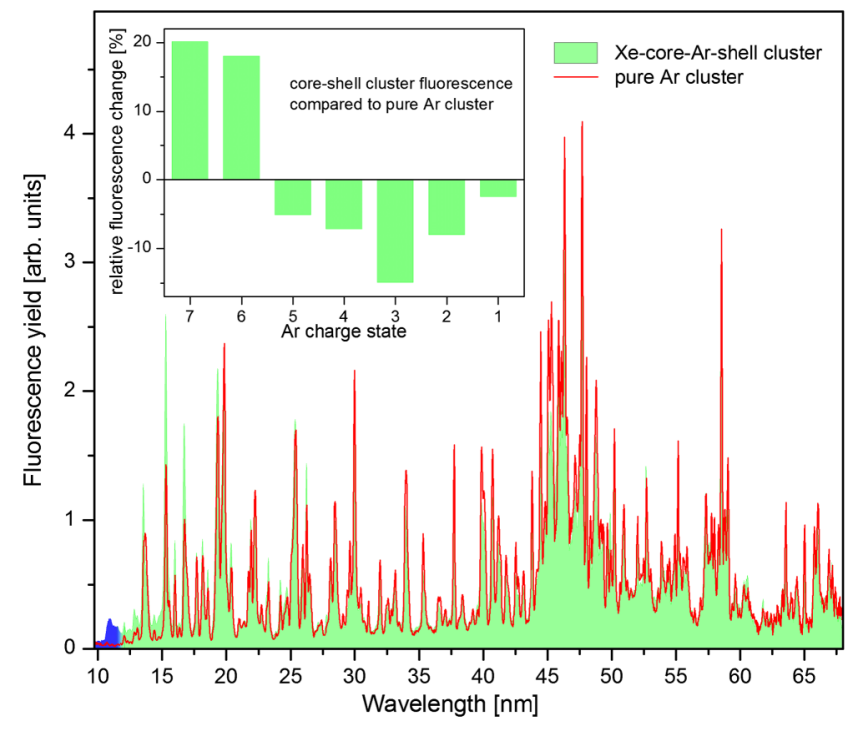

FIG. 3 (color online). Comparison of Xe-core-Ar-shell and pure Ar spectra. Relative changes of Ar line strength from coreshell clusters with respect to pure Ar clusters are shown in the inset. The distribution of multiply charged Ar ions localized in the shell is shifted toward higher charge states for core-shell clusters. Highly charged Xe ions localized in the nanoplasma core emit characteristic fluorescence at wavelength below $12 \mathrm{~nm}$ as indicated by the blue shaded area in the figure. An enlargement of this area is shown in Fig. 4

finding the core-shell spectrum is emulated by combining available spectra of pure Ar clusters (430 atoms) and pure $\mathrm{Xe}$ clusters (260 atoms). Whereas the Ar cluster is close to the size of the core-shell system the embedded Xe cluster is somewhat smaller. The spectral distribution of pure clusters exhibit only a weak dependence on the cluster size, though. In addition, a pure cluster shows different ionization and disintegration dynamics than a cluster core of the same size embedded in a larger shell. Therefore, we chose a larger Xe cluster as reference to account for the role of the Ar shell in the induced dynamics. The normalization factor is derived by an analysis of the elastic Rayleigh scattering signal in each spectrum. The resulting sum of the normalized Xe spectrum and the Ar spectrum is included in Fig. 4 in comparison to the measured spectra of core-shell clusters and pure Ar clusters. There is a clear similarity in the structures between 10.5 and $12 \mathrm{~nm}$ of the measured coreshell spectrum and the emulated one. We note that the interaction between the two constituents cannot be replicated by this method. Nevertheless, the result is clear evidence of the transient presence of highly charged $\mathrm{Xe}^{>10+}$ ions in the core-shell cluster. The Xe charge states observed in the core-shell cluster fluorescence are just as high as those in pure Xe spectra. This is a stark contrast to mass spectra, which detect much lower final charge states compared to pure clusters [27]. This proves that the signal must come from the cluster core. Furthermore, the detected plasma fluorescence must occur fast, before it is efficiently

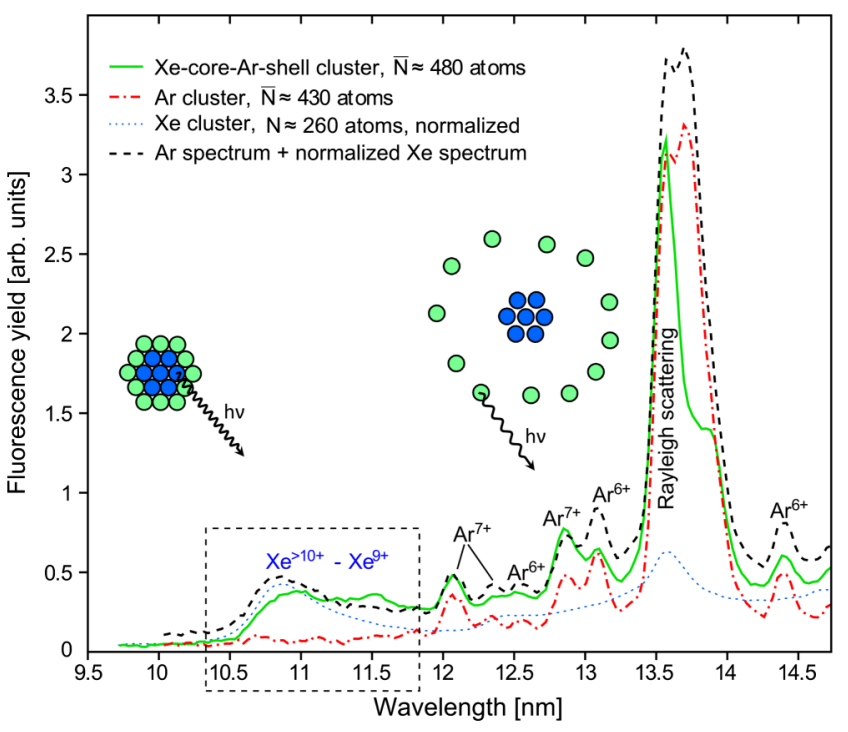

FIG. 4 (color online). Comparison of the measured Xe-coreAr-shell spectrum, the pure Ar and Xe spectra and the spectrum synthesized from the Ar- and a normalized Xe spectrum. The Xecore-Ar-shell spectrum and the synthesized spectrum show reasonable agreement at short wavelengths where Xe charge states of at least $11+$ appear. The pictographs illustrate that the time-integrated fluorescence spectrum of Xe-core-Ar-shell clusters provides information on the highly charged Xe core from early times of the interaction (left), as well as information on the Ar-shell upon Coulomb explosion (right).

suppressed in the center of the nanoplasma due to nonradiative recombination on a sub-ps time scale [28]. We note, that the Xe cluster core comprises only 80 ions which means that the core radius is doubled within 100-200 fs upon explosion [7] and the density of quasifree electrons, i.e., the recombination rate drops accordingly. If the highly charged Xe ions were still present after the cluster has significantly expanded, these charge states would also be present in the TOF ion spectra [27].

In summary, the ionization dynamics of Xe clusters coated with an Ar tamper layer exposed to FEL pulses at $10^{15} \mathrm{~W} / \mathrm{cm}^{2}$ has been studied with XUV fluorescence spectroscopy. The experiments give direct evidence thatin addition to the multiply charged Ar surface which Coulomb explodes and finally leads to fluorescence from individual ions-also the resonantly excited Xe core is initially highly charged. During the FEL interaction and before disintegration, Xe charge states above $q>10$ are generated and could clearly be identified by their characteristic radiative transitions. Therefore, XUV fluorescence spectroscopy probes ultrafast radiative decay of highly charged ions in a time window which is not accessible by conventional time-of-flight mass spectrometry. In agreement with theory, the latter traces remnants of the interaction, where electron thermalization followed by nonradiative recombination has already produced significantly lower charge states of mainly $\mathrm{Xe}^{+}$and $\mathrm{Xe}^{2+}$, 
respectively. Thus, a sacrificial tamper layer provides an efficient electron source for partial neutralization of highly charged ions created in the center. The significant reduction of charge states increases the available time for recording a diffraction pattern in coherent imaging experiments.

This research was supported by the Deutsche Forschungsgemeinschaft through Grants No. LA 1431/23, No. BO 3169/2-2, No. SFB925, and the excellence cluster "The Hamburg Centre for Ultrafast Imaging (CUI)." The experiments were carried out at the light source FLASH at DESY, a member of the Helmholtz Association (HGF). Finally, we would like to thank the whole FLASH team for their outstanding support.

*Corresponding author.tim.laarmann@desy.de

[1] W. Ackermann et al., Nat. Photonics 1, 336 (2007).

[2] P. Emma et al., Nat. Photonics 4, 641 (2010).

[3] T. Ishikawa et al., Nat. Photonics 6, 540 (2012).

[4] E. Allaria et al., New J. Phys. 14, 113009 (2012).

[5] R. Neutze, R. Wouts, D. van der Spoel, E. Weckert, and J. Hajdu, Nature (London) 406, 752 (2000).

[6] S. P. Hau-Riege et al., Phys. Rev. Lett. 104, 064801 (2010).

[7] T. Fennel, K.-H. Meiwes-Broer, J. Tiggesbäumker, P.-G. Reinhard, P. M. Dinh, and E. Suraud, Rev. Mod. Phys. 82, 1793 (2010).

[8] U. Saalmann, C. Siedschlag, and J. M. Rost, J. Phys. B 39, R39 (2006).

[9] T. Laarmann, K. von Haeften, H. Wabnitz, and T. Möller, J. Chem. Phys. 118, 3043 (2003).

[10] R. von Pietrowski, K. von Haeften, T. Laarmann, T. Möller, L. Museur, and A. V. Kanaev, Eur. Phys. J. D 38, 323 (2006).

[11] D. Rupp et al., New J. Phys. 14, 055016 (2012).

[12] Taking into account multistep ionization and the corresponding ionization potentials, the absorption of 17 photons is required to generate $\mathrm{Xe}^{11+}$ (see Fig. 2).
[13] U. Buck and R. Krohne, J. Chem. Phys. 105, 5408 (1996).

[14] M. Tchaplyguine, M. Lundwall, M. Gisselbrecht, G. Öhrwall, R. Feifel, S. Sorensen, S. Svensson, N. Mårtensson, and O. Björneholm, Phys. Rev. A 69, 031201 (2004).

[15] O. G. Danylchenko, Yu. S. Doronin, S. I. Kovalenko, M. Yu. Libin, V. N. Samovarov, and V. L. Vakula, Phys. Rev. A 76, 043202 (2007).

[16] S. Brühl, Ph.D. thesis, Universität Hamburg, 1996.

[17] N. Böwering, M. Martins, W. N. Partlo, and I. V. Fomenkov, J. Appl. Phys. 95, 16 (2004).

[18] A. Kramida et al., http://physics.nist.gov/asd.

[19] S. M. Vinko et al., Phys. Rev. Lett. 104, 225001 (2010).

[20] M. Arbeiter and T. Fennel, New J. Phys. 13, 053022 (2011).

[21] C. Gnodtke, U. Saalmann, and J. M. Rost, New J. Phys. 13, 013028 (2011).

[22] B. Ziaja, H. N. Chapman, R. Santra, T. Laarmann, E. Weckert, C. Bostedt, and T. Möller, Phys. Rev. A 84, 033201 (2011).

[23] C. Bostedt et al., Phys. Rev. Lett. 108, 093401 (2012).

[24] H. Thomas et al., Phys. Rev. Lett. 108, 133401 (2012).

[25] A. Sugishima et al., Phys. Rev. A 86, 033203 (2012).

[26] P. Di Cintio, U. Saalmann, and J. M. Rost, Phys. Rev. Lett. 111, 123401 (2013).

[27] M. Hoener, C. Bostedt, H. Thomas, L. Landt, E. Eremina, H. Wabnitz, T. Laarmann, R. Treusch, A. R. B. de Castro, and T. Möller, J. Phys. B 41, 181001 (2008).

[28] E. Ackad, N. Bigaouette, S. Mack, K. Popov, and L. Ramunno, New J. Phys. 15, 053047 (2013).

[29] M. Thomas, C. Bostedt, M. Hoener, E. Eremina, H. Wabnitz, T. Laarmann, E. Plönjes, R. Treusch, A. R. B. de Castro, and T. Möller, J. Phys. B 42, 134018 (2009).

[30] T. Fennel, L. Ramunno, and T. Brabec, Phys. Rev. Lett. 99, 233401 (2007).

[31] H. Iwayama, M. Nagasono, J. R. Harries, and E. Shigemasa, Opt. Express 20, 23174 (2012). 